\author{
KEIO UNIVERSITY \\ KEIO/KYOTO MARKET QUALITY RESEARCH PROJECT \\ (Global Center of Excellence Project)
}

KEIO/KYOTO GLOBAL COE DISCUSSION PAPER SERIES

DP2008-040

A Dynamic Game Model of Endogenous

Growth with Consumption Externalities

Katsuhiko Hori*

Akihisa Shibata**

\begin{abstract}
This paper introduces consumption externalities into an endogenous growth model of general capital accumulation and characterizes balanced growth equilibria. Contrary to the common argument in previous studies, we show that the growth rate in a feedback Nash equilibrium can be higher than that in an open-loop Nash equilibrium if agents strongly admire others' consumption.

*Katsuhiko Hori

GCOE Researcher, Institute of Economic Research, Kyoto University **Akihisa Shibata

Professor, Institute of Economic Research, Kyoto University
\end{abstract}

KEIO/KYOTO MARKET QUALITY RESEARCH PROJECT

(Global Center of Excellence Program)

Graduate School of Economics and Graduate School of Business and Commerce,

Keio University

2-15-45 Mita, Minato-ku, Tokyo 108-8345 Japan

Kyoto Institute of Economics,

Kyoto University

Yoshida-honmachi, Sakyo-ku, Kyoto 606-8501 Japan 


\title{
A Dynamic Game Model of Endogenous Growth with Consumption Externalities*
}

\author{
Katsuhiko Hori $^{\dagger} \quad$ Akihisa Shibata ${ }^{\ddagger}$
}

March 6, 2009

\begin{abstract}
This paper introduces consumption externalities into an endogenous growth model of general capital accumulation and characterizes balanced growth equilibria. Contrary to the common argument in previous studies, we show that the growth rate in a feedback Nash equilibrium can be higher than that in an open-loop Nash equilibrium if agents strongly admire others' consumption.
\end{abstract}

Keywords differential game; consumption externalities; endogenous growth; open-loop Nash equilibrium; feedback Nash equilibrium

${ }^{*}$ This work is supported by the Global Centre of Excellence (GCOE) program.

${ }^{\dagger}$ GCOE Researcher, Institute of Economic Research, Kyoto University, Yoshida, Sakyo-ku, Kyoto 606-8501, Japan

‡Professor, Institute of Economic Research, Kyoto University, Yoshida, Sakyo-ku, Kyoto 6068501, Japan 


\section{Introduction}

In the literature on commons games, it is usually argued that the lack of agents' commitment to their future actions leads to the so-called tragedy of the commons; that is, if agents condition their actions on the basis of the aggregate stock of the commons, rather than commit to their initial decisions, then it could worsen the overconsumption and/or underinvestment of the commons. ${ }^{1}$ For example, Fershtman and Nitzan (Ref. 2) develop a dynamic model of the voluntary provision of public goods and show that the contribution of conditioning agents to the collective contributions aggravates the degree of the free-rider problem ${ }^{2}$. Moreover, using endogenous growth models, Tornell and Velasco (Ref. 4) and Shibata (Ref. 5) show that the balanced growth rate without commitment is lower than that with commitment. ${ }^{3}$ This paper shows that this relationship between the growth rates with and without commitment is vulnerable; that is, if preferences include consumption externalities, the growth rate without commitment can be higher than that with commitment.

The existence of consumption externalities is emphasized in an early work by Veblen (Ref. 14) and validated as a determinant of aggregate consumption by Duesenberry (Ref. 15). Moreover, many recent studies analyze the effects of consumption externalities. For example, to reconcile the equity premium puzzle, Abel (Ref. 16), Constantinides (Ref. 17), and Galí (Ref. 18) incorporate the consumption externalities into consumption-based asset pricing models. Liu and Turnovsky (Ref. 19) examine the effects on the overconsumption phenomena in a growth model with consumption externalities. ${ }^{4}$ Along these lines of research, we construct a simple dynamic game model of common capital accumulation with consumption externalities and analyze how the presence of these externalities modifies the conventional results on the growth rates with and without commitment.

\section{The Model}

There are $N$ homogenous agents in our economy. They jointly produce a good by using common capital and divide it into consumption and common capital accumulation. The lifetime utility function of each agent is assumed to be additively

\footnotetext{
${ }^{1}$ Gordon (Ref. 1) is the first study that presents an example of the tragedy of the commons.

${ }^{2}$ See also Levhari and Mirman (Ref. 3).

${ }^{3}$ See also Benhabib and Radner (Ref. 6), Cozzi (Ref. 7), Dockner and Sorger (Ref. 8), Sorger (Ref. 9), Vencatachellum (Ref. 10), Vencatachellum (Ref. 11), Dockner and Nishimura (Ref. 12), and Luckraz (Ref. 13).

${ }^{4}$ See Ljungqvist and Uhlig (Ref. 20), Turnovsky and Monteiro (Ref. 21), and Mino (Ref. 22) for other recent examples.
} 
separable in time. The agent's subjective discount rate is denoted by $\rho$ and the elasticity of intertemporal substitution is represented by $\eta$. We specify the lifetime utility function as follows: ${ }^{5}$

$$
\bar{U}_{i}=\int_{0}^{\infty} \frac{\eta}{\eta-1}\left(c_{i} \bar{c}_{i}^{-\alpha}\right)^{1-\frac{1}{\eta}} \exp (-\rho t) \mathrm{d} t \quad \text { for } i=1, \ldots, N,
$$

where $c_{i}: \mathbb{R}_{+} \rightarrow \mathbb{R}_{+}$is countinuously differentiable; $\bar{c}_{i}=\sum_{j \neq i} c_{j} /(N-1) ; \rho, \eta \in$ $\mathbb{R}_{++}$; and $\alpha \in(-\infty, 1)$. Here, $c_{i}$ is the consumption of agent $i$ and $\bar{c}_{i}$ is the level of average consumption of other agents, which represents the externalities from other agents' consumption. Parameter $\alpha$ represents the magnitude of the external effects of consumption. Following Dupor and Liu (Ref. 23), we define "jealousy" and "admiration" as follows.

Definition 2.1. We say that consumption externalities indicate

1. "jealousy" if $\alpha>0$ while "admiration" if $\alpha<0$.

2. "keeping up with the Joneses" (KUJ) if $\alpha(1-\eta)>0$ and "running away from the Joneses" $(R A J)$ if $\alpha(1-\eta)<0$.

The definitions of jealousy and admiration are intuitively interpreted as follows. If the utility of an agent decreases as others' consumption rises, we can state that his preference exhibits jealousy. On the other hand, if the utility increases as others' consumption falls, we state that the preference exhibits admiration. Similarly, if the marginal utility increases as others' consumption rises, we state that his preference exhibits KUJ, and, if not we state that the preference exhibits RAJ.

The production technology is defined as of the $A k$ form, and therefore, the dynamics of the common capital stock are

$$
\dot{k}=A k-\sum_{i=1}^{N} c_{i}, \quad \text { given } k_{0},
$$

where $k: \mathbb{R}_{+} \rightarrow \mathbb{R}_{+}, k_{0} \in \mathbb{R}_{++}$, and $A \in \mathbb{R}_{++}$. Here, $k$ denotes the stock of common capital, $k_{0}$ is its initial value, and $A$ is a constant productivity parameter. If each agent chooses a symmetric strategy, we have $c=c_{i}$ for all $i=1, \cdots, N$. In this case, from (2), the growth rate, $g$, can be written as

$$
g=A-N \frac{c}{k}
$$

Throughout this paper, we assume the following condition.

\footnotetext{
${ }^{5}$ This specification follows Galí (Ref. 18).
} 
Assumption 2.1. It holds that

$$
\psi<\frac{\rho}{A}<\min \left(1, \frac{1-\alpha}{N}\right),
$$

where $\psi=(1-\alpha)(1-1 / \eta)$.

Here, we impose Assumption 2.1 to ensure that the problem is well defined and that the rate of balanced growth is positive. ${ }^{6}$

\section{Open-loop Nash Equilibrium}

We first consider a situation where agents commit to their announced actions.

Definition 3.1 (Open-loop Nash equilibrium). An N-tuple of consumption paths, $\left(c_{1}, \ldots, c_{N}\right)$, is called an open-loop Nash equilibrium if, for each $i=1, \ldots, N, c_{i}$ maximizes (1) subject to (2).

An open-loop Nash equilibrium is a plausible equation concept in the situation where agents can not observe the value of common capital at each time ${ }^{7}$, and thus, condition their strategies only on the basis of the initial value of common capital, and precommit themselves to their future consumption paths. To solve the problem, we define the following current value Hamiltonian:

$$
\mathscr{H}_{i}=\frac{\eta}{\eta-1}\left(c_{i} \bar{c}_{i}^{-\alpha}\right)^{1-\frac{1}{\eta}}+q_{i}^{o}\left(A k-\sum_{i=1}^{N} c_{i}\right),
$$

where $q_{i}^{o}$ is the costate variable of the common capital. The optimal conditions for this problem are given as

$$
\begin{aligned}
& c_{i}^{-\frac{1}{\eta}} \bar{c}_{i}^{-\alpha\left(1-\frac{1}{\eta}\right)}=q_{i}^{o}, \\
& A q_{i}^{o}=\rho q_{i}^{o}-\dot{q}_{i}^{o},
\end{aligned}
$$

and

$$
\lim _{t \rightarrow \infty} q_{i}^{o} k \exp (-\rho t)=0
$$

\footnotetext{
${ }^{6}$ By Assumption 2.1, the value function of each agent is well defined. Some literature studies the case that it does not exist in finite values and refers to the several concepts of optimality. See, for example, studies such as Stern (Ref. 24); Seierstad and Sydsæter (Ref. 25); Dockner, Jorgensen, van Long, and Sorger (Ref. 26).

${ }^{7}$ For example, if agents have to pay extremely high value of cost to observe the current amount of instracture accumulated in the economy, they would give up to observe the current amount of infrastracture and adopt the open-loop Nash equilibrium.
} 
In equilibrium, it follows from (5) and (6) that $c=\bar{c}_{i}=c_{i}$ for all $i=1, \cdots, N$, and thus, the Euler equation is given as

$$
\frac{\dot{c}}{c}=\frac{A-\rho}{1-\psi} .
$$

Therefore, we have the following result.

Proposition 3.1. The growth rate in the open-loop Nash equilibrium is given by

$$
g^{o}=\frac{A-\rho}{1-\psi}
$$

Proof. From (3), (5), and (6), we obtain the balanced growth rate in this open-loop Nash equilibrium.

Note that from conditions (5) to (7), we have a unique open-loop Nash equilibrium in this model. It is also easy to show that the open-loop Nash equilibrium is Pareto efficient. This efficiency result is similar to that derived by Chiarella, Kemp, van Long, and Okuguchi (Ref. 27), who show, contrary to the traditional view, that an open-loop Nash equilibrium can be socially efficient in various situations.

\section{Feedback Nash Equilibrium}

We next derive the case where each agent cannot commit to his future actions. This case can be analyzed by applying the feedback Nash equilibrium concept, which allows agents to choose and expect optimal consumption paths that depend on the current stock of common capital at each time.

Definition 4.1 (Feedback Nash equilibrium). An N-tuple of consumption paths, $\left(c_{1}, \ldots, c_{N}\right)$, is called a feedback Nash equilibrium if, for each $i=1, \ldots, N, c_{i}$ maximizes (1) subject to (2) and $c_{j}=c_{j}(k)$ for $j \neq i$.

Note that the feedback Nash equilibrium implies that agents can monitor the stock of common capital, and they condition their future consumption paths on the value of common capital at each time. We solve the feedback Nash equilibrium by using dynamic programming. The Hamilton-Jacobi-Bellman equation of this problem is

$$
\rho U_{i}(k)=\max _{c_{i}}\left[\frac{\eta}{\eta-1}\left(c_{i} \bar{c}_{i}(k)^{-\alpha}\right)^{1-\frac{1}{\eta}}+q_{i}^{f}\left(A k-\sum_{i=1}^{N} c_{i}\right)\right],
$$


where $\bar{c}_{i}(k)=\sum_{j \neq i} c_{j}(k) /(N-1) ; q_{i}^{f}=d U_{i} / d k$; and $U_{i}(k)$, defined by below, denotes the value function of agent $i$.

$$
U_{i}(k)=\max \int_{t}^{\infty} \frac{\eta}{\eta-1}\left(c_{i} \bar{c}_{i}(k)^{-\alpha}\right)^{1-\frac{1}{\eta}} \exp (-\rho(s-t)) \mathrm{d} s .
$$

From (10), the first-order condition is obtained as

$$
c_{i}^{-\frac{1}{\eta}} \bar{c}_{i}(k)^{-\alpha\left(1-\frac{1}{\eta}\right)}=q_{i}^{f}(k) .
$$

In what follows, we consider the equilibrium in linear and nonlinear strategies.

\subsection{Equilibrium in Linear Strategies}

In this subsection, we consider equilibrium where the agents asopt the following symmetric linear strategy.

$$
c(k)=\beta k+\gamma,
$$

where $\beta$ and $\gamma$ are constants. In this situation, we have the following lemma:

Lemma 4.1. If agents adopt the linear strategy defined in (13), it holds that

$$
\omega_{B G P}=\frac{\rho-\psi A}{1-\alpha-\psi N} \quad \text { for all } t \in \mathbb{R}_{+},
$$

and

$$
U(k)=\frac{c(k)^{\psi}}{\psi \omega_{B G P}} \quad \text { for all } k \in \mathbb{R}_{+}
$$

where $\omega_{B G P}$ denotes the ratio of consumption to common capital in the case that the agents adopt the linear strategy.

Proof. From (12) it holds that in equilibrium,

$$
U(k)=\frac{1}{\beta \psi} c(k)^{\psi}+\delta,
$$

where $\delta$ denotes an integral constant. By using (12) and (16), (11) can be written as

$$
\frac{\rho}{\beta \psi} c(k)^{\psi}+\delta=\left(\frac{\eta}{\eta-1}-N+A \frac{k}{\beta k+\gamma}\right) c(k)^{\psi} .
$$


Therefore, for the above equality to hold for any value of $k$, it must hold that

$$
\delta=\gamma=0
$$

and

$$
\beta=\frac{\rho-\psi A}{1-\alpha-\psi N}
$$

Thus, by substituting (17) and (18) into (13) and (16), we have (14) and (15), respectively.

From Lemma 4.1, we have the following proposition.

Proposition 4.1. If agents adopt the linear strategy defined in (13), the growth rate in the feedback Nash equilibrium is positive and given as

$$
g_{B G P}^{f}=N\left(\omega_{S S}-\omega_{B G P}\right),
$$

where $g_{B G P}^{f}$ denotes the growth rate of the economy and $\omega_{S S}=A / N$.

Proof. If agents adopt (13), Lemma 4.1 holds. Therefore, substituting (14) into (3), we get

$$
\frac{\dot{k}}{k}=\frac{\dot{c}}{c}=A+\frac{N(\psi A-\rho)}{1-\alpha-\psi N}=N\left(\omega_{\mathrm{SS}}-\omega_{\mathrm{BGP}}\right),
$$

where $\omega_{\mathrm{SS}}>\omega_{\mathrm{BGP}}$ by Assumption 2.1. Next we check that (19) satisfies the transversality condition. From (15) and (19), we get

$$
U(k)=\frac{c_{0}^{\psi}}{\psi \omega_{\mathrm{BGP}}} \exp \left(\frac{\psi[A(1-\alpha)-\rho N]}{1-\alpha-\psi N} t\right) .
$$

Therefore, we have

$$
\lim _{t \rightarrow \infty} U(k) \exp (-\rho t)=\lim _{t \rightarrow \infty} \frac{c_{0}^{\psi}}{\psi \omega_{\mathrm{BGP}}} \exp \left((\alpha-1) g_{\mathrm{BGP}}^{f} t\right)=0
$$

since $\alpha<1$ and $g_{\mathrm{BGP}}^{f}>0$ by the assumptions. 


\subsection{Equilibrium in Nonlinear Strategies}

This subsection considers a more general case where agents adopt symmetric nonlinear strategies. ${ }^{8}$

Lemma 4.2. In a symmetric feedback Nash equilibrium, it holds that

$$
k=Z c^{\frac{A}{A-\rho}(1-\psi)}+\frac{c}{\omega_{B G P}},
$$

where $Z \in \mathbb{R}$ denotes an integral constant.

Proof. In a symmetric equilibrium, it follows from (12) that (10) can be written as

$$
\rho U=\left(\frac{\eta}{\eta-1}-N\right) q^{f \frac{\psi}{\psi-1}}+q^{f} A k .
$$

Here, note that from (4), the coefficient of the first term on the right-hand side of the above equation is positive. Therefore, since (22) is D'Alembert's (or Lagrange's) equation, the solution of (22) satisfies the following equation:

$$
k=Z q^{f^{-\frac{A}{A-\rho}}}+\frac{1-\alpha-\psi N}{\rho-\psi A} q^{f \frac{1}{\psi-1}},
$$

Again, using (12), we get (21).

The sign of $Z$ in Lemma 4.2 plays a crucial role in characterizing the dynamics of the economy, as will be clear in the statement in the last part of this section. Lemma 4.2 gives the dynamics of the economy as follows.

Proposition 4.2. The dynamics of consumption in a symmetric feedback Nash equilibrium are characterized by (3) and

$$
\frac{\dot{c}}{c}=\frac{N(A-\rho)\left(\omega_{S S}-\omega\right)}{(1-\alpha-\psi N)\left(\omega_{U B}-\omega\right)},
$$

where $\omega=c / k$ and

$$
\omega_{U B}=\frac{(1-\psi) A}{1-\alpha-\psi N}
$$

\footnotetext{
${ }^{8}$ See, for example, Tsutsui and Mino (Ref. 28), Dockner and Sorger (Ref. 8), Sorger (Ref. 9), Vencatachellum (Ref. 11), and Itaya and Shimomura (Ref. 29).
} 
Proof. Since $\omega=\omega_{\mathrm{UB}}$ is not feasible since it violates the differentiability of $c$, we assume that $\omega \neq \omega_{\mathrm{UB}}$ in the following analyses. From Lemma 4.2 , in the symmetric feedback Nash equilibrium, we have (21). Rearranging (21) yields

$$
Z=\left(k-\frac{c}{\omega_{\mathrm{BGP}}}\right) c^{\frac{A}{A-\rho}(\psi-1)} .
$$

Taking the logarithm of both sides of the above equation and differentiating them with respect to $t$, we have

$$
\frac{\dot{k}}{k}=\left[(1-\alpha-\psi N) \frac{c}{k}+(1-\psi) A\right] \frac{1}{A-\rho} \frac{\dot{c}}{c} .
$$

Therefore, by substituting (3) into the above equation, we have (23).

Note that (23) is a necessary condition for the symmetric feedback Nash equilibrium, since it is derived from the first-order condition (12).

Before we state Proposition 4.3, we state the following lemma.

Lemma 4.3. In the symmetric feedback Nash equilibrium, it holds that

$$
\dot{\omega}=\frac{N\left(\omega_{B G P}-\omega\right)\left(\omega-\omega_{S S}\right) \omega}{\omega_{U B}-\omega} .
$$

Proof. Since $\omega=\omega_{\mathrm{UB}}$ is not feasible, we assume here that $\omega \neq \omega_{\mathrm{UB}}$. Subtracting (3) from (23), we have

$$
\frac{\dot{\omega}}{\omega}=\frac{\dot{c}}{c}-\frac{\dot{k}}{k}=\frac{N\left(\omega_{\mathrm{BGP}}-\omega\right)\left(\omega-\omega_{\mathrm{SS}}\right)}{\omega_{\mathrm{UB}}-\omega} .
$$

The above equation completes the proof.

Therefore, the dynamics of the economy are summarized as that of $\omega$. We now state the following proposition.

Proposition 4.3. Denoting the growth rate of consumption in the feedback Nash equilibrium by $g_{c}$, we have the following:

1. In the case that $\omega_{0}=\omega_{B G P}$, the growth rate in the feedback Nash equilibrium is given by (19).

2. In the case that $\omega_{0}>\omega_{B G P}$, the feedback Nash equilibria are only the steady states if $1-\alpha>N$ and they converge to steady states if $1-\alpha<N: g_{c}^{f} \rightarrow 0$ as $t \rightarrow \infty$. 
3. In the case that $\omega<\omega_{B G P}$, the growth rate in the feedback Nash equilibrium converges to that in the open-loop Nash equilibrium (9): $g_{c}^{f} \rightarrow g^{o}$ as $t \rightarrow \infty$, where $\omega_{0}$ denotes the initial ratio of $c$ to $k: \omega=\omega_{0}$ at $t=0$.

Proof. 1. Case 1: $\omega_{0}=\omega_{\mathrm{BGP}}$ Substituting $\omega_{0}=\omega_{\mathrm{BGP}}$ into (3), we have the result.

2. Case 2: $\omega_{0}>\omega_{\mathrm{BGP}}$ See Appendix.

3. Case 3: $\omega_{0}<\omega_{\mathrm{BGP}}$ It follows from (24) that $\dot{\omega}<0$ for all $\omega<\omega_{\mathrm{BGP}}$ and that $\dot{\omega}=0$ at $\omega=0$. Since this implies $\omega \rightarrow 0$ as $t \rightarrow \infty$, Proposition 4.2 gives

$$
\lim _{t \rightarrow \infty} \frac{\dot{c}}{c}=\frac{N(A-\rho) \omega_{\mathrm{SS}}}{(1-\alpha-\psi N) \omega_{\mathrm{UB}}}=\frac{A-\rho}{1-\psi},
$$

where the right-hand side is the same as the growth rate in the open-loop Nash equilibrium. Therefore, it satisfies the transversality condition under Assumption 2.1.

Figure 1 is the phase diagram in the case that $1-\alpha<N$. The solid curves in Figure 1 depict the loci of capital and consumption satisfying (21) corresponding to various values of $\omega_{0}$ in Proposition 4.2. The BGP line plots the locus of capital and consumption in the case that $\omega_{0}=\omega_{\mathrm{BGP}}$, and the curves above and below the BGP line depict the loci in the case that $\omega_{0}>\omega_{\mathrm{BGP}}$ and $\omega_{0}<\omega_{\mathrm{BGP}}$, respectively. The SS line depicts the $\dot{k}=0\left(\omega=\omega_{\mathrm{SS}}\right)$ locus, and thus, $\dot{k}>0$ when a path is located below the line and $k<0$ when it lies in the area above the line. The slope of the SS line is larger than that of the BGP line by Assumption 2.1. It should also be noted that the SS line in the case that $\omega_{0}>\omega_{\mathrm{BGP}}$ lies in the area below the UB line. The UB line plots $\omega=\omega_{\mathrm{UB}}$, at which the slope of the peaks of the curves drawn from (21) become infinite.

The case that $\omega_{0}=\omega_{\mathrm{BGP}}$ corresponds to the balanced growth path. Note that a balanced growth is realized only when $\omega_{0}=\omega_{\text {BGP. }}$. Here, it should be noted that the growth rate in this case coincides with (19), and thus, it satisfies the transversality condition.

In the case that $\omega_{0}>\omega_{\mathrm{BGP}}$, since the paths in the area above the UB line move up in the north-west direction along the curves drawn from (21) and reach the vertical axis in a finite period, the equilibrium paths drawn from (21) must lie between the UB and BGP lines. Moreover, since the paths between the UB and SS line move down in the south-west direction and the paths between the SS and 


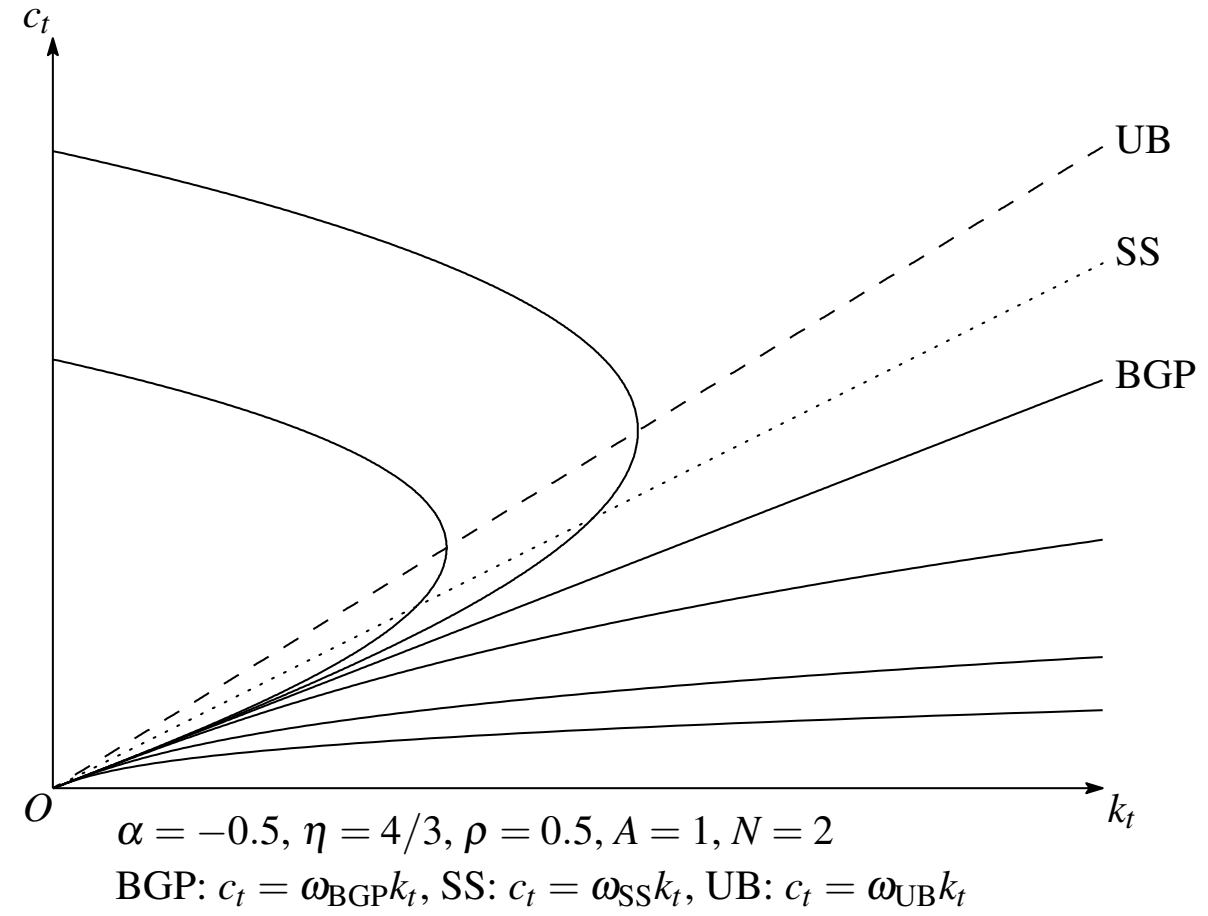

Figure 1: Dynamics for feedback Nash equilibrium 
BGP line move up in the north-east direction along the curves, the equilibrium paths converge to the SS line. This implies there are infinitely many feedback Nash equilibria which converge to corresponding steady states. ${ }^{9}$

In the case that $\omega_{0}<\omega_{\mathrm{BGP}}$, the paths must be below the BGP line and move up in the north-east direction along the curves. Along these curves, the growth rates converge to the balanced growth path in the open-loop Nash equilibrium. Therefore, we find that there also exist infinitely many nonbalanced growth paths in the feedback Nash equilibrium.${ }^{10}$

\section{Comparison of the Growth Rates in Open-loop and Feedback Nash Equilibria}

\subsection{Linear Strategies}

As Tornell and Velasco (Ref. 4) and Shibata (Ref. 5) illlustrate, in an economy without consumption externalities, the balanced growth rate in the feedback Nash equilibrium is lower than that in the open-loop Nash equilibrium. In this section, we show that the existence of consumption externalities may destroy this relationship between the two equilibrium growth rates.

Theorem 5.1. Under Assumption 2.1, it holds that

$$
g_{B G P}^{f}>g^{o}
$$

if and only if

$$
\alpha<1-N \text {. }
$$

Proof. To compare the growth rates in the open-loop and feedback Nash equilibria, presented in respectively, (9) and (19), we calculate their difference:

$$
g_{\mathrm{BGP}}^{f}-g^{o}=\frac{\omega_{\mathrm{BGP}}}{1-\psi}(1-\alpha-N) .
$$

Since the first multiplying term is positive by Assumption 2.1, the growth rate in the feedback Nash equilibrium becomes higher than that in the open-loop Nash equilibrium if and only if (25) holds.

\footnotetext{
${ }^{9}$ Note that these equilibrium paths are not Markov perfect since the domain of each path does not span the entire state space. However, we can easily extend the strategy to be made Markov perfect. For example, by extending the strategy to (21) with $\omega>\omega_{\mathrm{BGP}}$ if $k>k_{\max }$, where $k_{\max }$ denotes a maximum value of $k$ satisfying (21), it can be Markov perfect. See also Clemhout and Wan Jr. (Ref. 30) and Shibata (Ref. 5).

${ }^{10}$ See also Shibata (Ref. 5).
} 
Note that under (25), we can always choose the value of $\eta$ to satisfy (4); that is, we can prove that in this model, there exists a situation in which (26) is positive. Since $N>1$, (25) states that the growth rate in the feedback Nash equilibrium is higher than that in the open-loop Nash equilibrium if preferences exhibit admiration to other agents' consumption. This result shows that although the growth rate in the feedback Nash equilibrium is lower than that in the open-loop Nash equilibrium as generally argued, the presence of strong admiration reverses the conclusion.

Intuitively, the presence of jealousy implies that the utility of agents decreases with an increase of the others' consumption. This suggests that the presence of jealousy is a factor raising their current consumption and reducing their contribution to the accumulation of common capital. On the other hand, the presence of admiration implies that their utility increases with increase of the others' consumption, suggesting that admiration is a factor raising contribution to the accumulation of common capital. It shows that the growth rate in the feedback Nash equilibrium, where agents can change their consumption on the basis of others' consumption, may be higher than that in the open-loop Nash equilibrium if the degree of admiration is strong enough. Alternatively, the presence of admiration can also be interpreted as the other source of the free-rider problem, since they can enjoy utility from others' consumption. In this sense, it can be said that the growth rate without commitment becomes higher than that with commitment if the free ride on others' consumption is stronger than that on the common capital accumulation.

Finally, we make the following remark.

Remark 5.1. The relative magnitude of the two growth rates is irrelevant to whether preferences exhibit KUJ $(\alpha(1-\eta)>0)$ or $R A J(\alpha(1-\eta)<0)$.

Remark 5.1 follows from the fact that (25) does not include the intertemporal elasticity of substitution.

\subsection{Nonlinear Strategies}

Finally, we compare the open-loop Nash equilibrium and the nonlinear feedback Nash equilibrium. It is obvious that the growth rate in the open-loop Nash equilibrium is higher than that in the feedback Nash equilibrium in the long run in the case that $Z<0$, where consumption and capital stock converge to a steady state. In the case that $Z>0$, the growth rate of common capital converges to $A$ since $\omega$ converges to 0 . We have the following theorem on the rate of consumption growth. 
Theorem 5.2. There exist infinitely many nonlinear feedback Nash equilibria such that

$$
g_{c}^{f}>g^{o} \quad \text { for all } t \in \mathbb{R}_{+}
$$

if and only if $\alpha<1-N$ and $\omega_{0}<\omega_{B G P}$.

Proof. Rearranging (23), we have

$$
g_{c}^{f}=\frac{A-\rho}{1-\psi} \frac{(1-\psi) A-(1-\psi) N \omega}{(1-\psi) A-(1-\alpha-\psi N) \omega} .
$$

The above equation implies that $g_{c}^{f}>g^{o}$ if $(1-\psi) N<(1-\alpha-\psi N)$, that is, $\alpha<1-N$.

Therefore, we find the same condition for the ordinal relationship between growth rates to be destroyed as in the case that agents adopt linear feedback strategies, (25), even if they adopt nonlinear ones.

\section{Conclusion}

This paper introduced consumption externalities into a dynamic game growth model of common capital accumulation. Contrary to the general argument that the growth rate in a feedback Nash equilibrium is lower than that in an open-loop Nash equilibrium, we showed that in our model, the growth rate in the feedback Nash equilibrium under both linear and nonlinear strategies could be higher than that in the open-loop Nash equilibrium if agents strongly admire others' consumption, while a conventional relationship is maintained between the two growth rates if the agents envy others' consumption. 


\section{Appendix}

\section{Proof of Proposition 4.3 in the Case that $\omega_{0}>\omega_{\mathrm{BGP}}$}

We first consider the case that $1-\alpha>N$. Noting that $\omega_{\mathrm{SS}}>\omega_{\mathrm{UB}}>\omega_{\mathrm{BGP}}$ in this case, we investigate (24) by classifying four cases.

1. $\omega_{0}>\omega_{\mathrm{SS}}$

In this case, it must hold that

$$
\dot{\omega} \geq \dot{\omega}_{0}>0,
$$

and thus, it follows from (3) that

$$
\frac{\dot{k}}{k} \leq A-N \omega_{0}<0 .
$$

Therefore, the value of $k$ reaches 0 in finite time. Since this implies that (21) cannot hold for any $c \in \mathbb{R}_{+}$at some finite time, we do not classify this case as feedback Nash equilibrium.

2. $\omega_{\mathrm{SS}}=\omega_{0}$

In this case, we have

$$
\dot{\omega}=\dot{k}=\dot{c}=0 \quad \text { for all } t \in \mathbb{R}_{+} .
$$

Since this case obviously satisfies the transversality condition and (21) for all $t \in$ $\mathbb{R}_{+}$, it must be classified as a feedback Nash equilibrium.

3. $\omega_{\mathrm{SS}}>\omega_{0}>\omega_{\mathrm{UB}}$

In this case, it follows that

$$
\dot{\omega}<\frac{N\left(\omega_{\mathrm{UB}}-\omega_{\mathrm{BGP}}\right)\left(\omega_{\mathrm{SS}}-\omega_{0}\right) \omega_{\mathrm{UB}}}{\omega_{\mathrm{UB}}-\omega_{0}}<0 .
$$

Therefore, the value of $\omega$ reaches $\omega_{\mathrm{UB}}$ in finite time, where the right-hand side of (24) diverges and (21) is not satisfied.

4. $\omega_{\mathrm{UB}}>\omega_{0}>\omega_{\mathrm{BGP}}$

In this case, it holds that

$$
\dot{\omega}>\frac{N\left(\omega_{0}-\omega_{\mathrm{BGP}}\right)\left(\omega_{\mathrm{SS}}-\omega_{\mathrm{UB}}\right) \omega_{\mathrm{BGP}}}{\omega_{\mathrm{UB}}-\omega_{0}}>0 .
$$

Therefore, the value of $\omega$ reaches $\omega_{\mathrm{UB}}$ in finite time, where the right-hand side of (24) diverges and (21) does not hold. Thus, it cannot be classified as a feedback Nash equilibrium.

Therefore, in the case that $1-\alpha>N$, we have only the steady states as feedback Nash equilibria.

We next consider the case that $1-\alpha<N$. In this case, $\omega_{\mathrm{UB}}>\omega_{\mathrm{SS}}>\omega_{\mathrm{BGP}}$ by Assumption 2.1 . We investigate (24) by classifying two cases. 
1. $\omega_{0}>\omega_{\mathrm{UB}}$

In this case, it holds that

$$
\dot{\omega} \geq \dot{\omega}_{0}>0
$$

and thus, it follows from (3) that

$$
\frac{\dot{k}}{k} \leq A-N \omega_{0}<0 .
$$

Therefore, the value of $k$ reaches 0 in finite time. Since this implies that (21) cannot hold for any $c \in \mathbb{R}_{+}$at some finite time, we exclude this case as a feedback Nash equilibrium.

2. $\omega_{\mathrm{UB}}>\omega_{0}>\omega_{\mathrm{BGP}}$

In this case,

$$
\dot{\omega}<0 \quad \text { if } \omega_{0}>\omega_{\mathrm{SS}}
$$

and

$$
\dot{\omega}>0 \quad \text { if } \omega_{0}<\omega_{\mathrm{SS}} .
$$

Therefore, $\omega \rightarrow \omega_{\text {SS }}$ as $t \rightarrow \infty$, where

$$
\dot{\omega}=\dot{k}=\dot{c}=0 .
$$

The transversality condition is obviously satisfied in this case. 


\section{References}

1. H.S. Gordon. The Economic Theory of a Common Property Resource. Journal of Political Economy, 62(2):124-142, 1954.

2. C. Fershtman and S. Nitzan. Dynamic Voluntary Provision of Public Goods. European Economic Review, 35:1057-1067, 1991.

3. D. Levhari and L.J. Mirman. The Great Fish War: An Example Using a Dynamic Cournot-Nash Solution. The Bell Journal of Economics, 11(1):322334, 1980.

4. A. Tornell and A. Velasco. The Tragedy of the Commons and Economic Growth: Why Does Capital Flow from Poor to Rich Countries? The Journal of Political Economy, 100(6):1208-1231, 1992.

5. A. Shibata. Strategic Interactions in a Growth Model with Infrastructure Capital. Metroeconomica, 53(4):434-460, 2002.

6. J. Benhabib and R. Radner. The Joint Exploitation of a Productive Asset: A Game-Theoretic Approach. Economic Theory, 2(2):155-190, 1992.

7. G. Cozzi. R\&D Cooperation and Growth. Journal of Economic Theory, 86 (1):17-49, 1999.

8. E.J. Dockner and G. Sorger. Existence and Properties of Equilibria for a Dynamic Game on Productive Assets. Journal of Economic Theory, 71(1): 209-227, 1996.

9. G. Sorger. Markov-Perfect Nash Equilibria in a Class of Resource Games. Economic Theory, 11(1):79-100, 1998.

10. D. Vencatachellum. Endogenous Growth with Strategic Interactions. Journal of Economic Dynamics and Control, 23(2):233-254, 1998.

11. D. Vencatachellum. A Differential R\&D Game: Implications for KnowledgeBased Growth Models. Journal of Optimization Theory and Applications, 96 (1):175-189, 1998.

12. E.J. Dockner and K. Nishimura. Capital Accumulation Games with a Nonconcave Production Function. Journal of Economic Behavior and Organization, 57(4):408-420, 2005.

13. S. Luckraz. Process Spillovers and Growth. Journal of Optimization Theory and Applications, 2008. online first. 
14. T. Veblen. The Theory of the Leisure Class. Macmillan, New York, 1912.

15. J.S. Duesenberry. Income, Saving, and the Theory of Consumer Behavior. Harvard University Press, Cambridge, MA, 1949.

16. A.B. Abel. Asset Prices under Habit Formation and Catching Up with the Joneses. The American Economic Review, 80(2):38-42, 1990.

17. G.M. Constantinides. Habit Formation: A Resolution of the Equity Premium Puzzle. The Journal of Political Economy, 98(3):519-543, 1990.

18. J. Galí. Keeping Up with the Joneses: Consumption Externalities, Portfolio Choice, and Asset Prices. Journal of Money, Credit \& Banking, 26(1):1-8, 1994.

19. W.F. Liu and S.J. Turnovsky. Consumption Externalities, Production Externalities, and Long-Run Macroeconomic Efficiency. Journal of Public Economics, 89(5-6):1097-1129, 2005.

20. L. Ljungqvist and H. Uhlig. Tax Policy and Aggregate Demand Management under Catching Up with the Joneses. The American Economic Review, 90(3): 356-366, 2000.

21. S.J. Turnovsky and G. Monteiro. Consumption Externalities, Production Externalities, and Efficient Capital Accumulation under Time Non-separable Preferences. European Economic Review, 51(2):479-504, 2007.

22. K. Mino. Growth and Bubbles with Consumption Externalities. Japanese Economic Review. forthcoming.

23. B. Dupor and W.F. Liu. Jealousy and Equilibrium Overconsumption. The American Economic Review, 93(1):423-428, 2003.

24. Le Stern. Criteria of Optimality in the Infinite-Time Optimal Control Problem. Journal of Optimization Theory and Applications, 44(3):497-508, 1984.

25. A. Seierstad and K. Sydsæter. Optimal Control Theory with Economic Applications. Advanced Textbooks in Economics. North Holland, 3 edition, 1987.

26. E.J. Dockner, S. Jorgensen, N. van Long, and G. Sorger. Differential Games in Economics and Management Science. Cambridge University Press, 2000.

27. C. Chiarella, M.C. Kemp, N. van Long, and K. Okuguchi. On the Economics of International Fisheries. International Economic Review, 25(1): 85-92, 1984. 
28. S. Tsutsui and K. Mino. Nonlinear Strategies in Dynamic Duopolistic Competition with Sticky Prices. Journal of Economic Theory, 52(1):136-161, 1990.

29. J. Itaya and K. Shimomura. A Dynamic Conjectural Variations Model in the Private Provision of Public Goods: A Differential Game Approach. Journal of Public Economics, 81(1):153-172, 2001.

30. S. Clemhout and H.Y. Wan Jr. Endogenous Growth as a Dynamic Game. Birkhauser, Boston, MA, 1996. 